This item was submitted to Loughborough's Research Repository by the author.

Items in Figshare are protected by copyright, with all rights reserved, unless otherwise indicated.

\title{
A novel single lag auto-correlation minimization (SLAM) algorithm for blind adaptive channel shortening
}

PLEASE CITE THE PUBLISHED VERSION

PUBLISHER

(c) IEEE

VERSION

VoR (Version of Record)

\section{LICENCE}

CC BY-NC-ND 4.0

\section{REPOSITORY RECORD}

Nawaz, Rab, and Jonathon Chambers. 2019. "A Novel Single Lag Auto-correlation Minimization (SLAM) Algorithm for Blind Adaptive Channel Shortening". figshare. https://hdl.handle.net/2134/5666. 
This item was submitted to Loughborough's Institutional Repository (https://dspace.lboro.ac.uk/) by the author and is made available under the following Creative Commons Licence conditions.

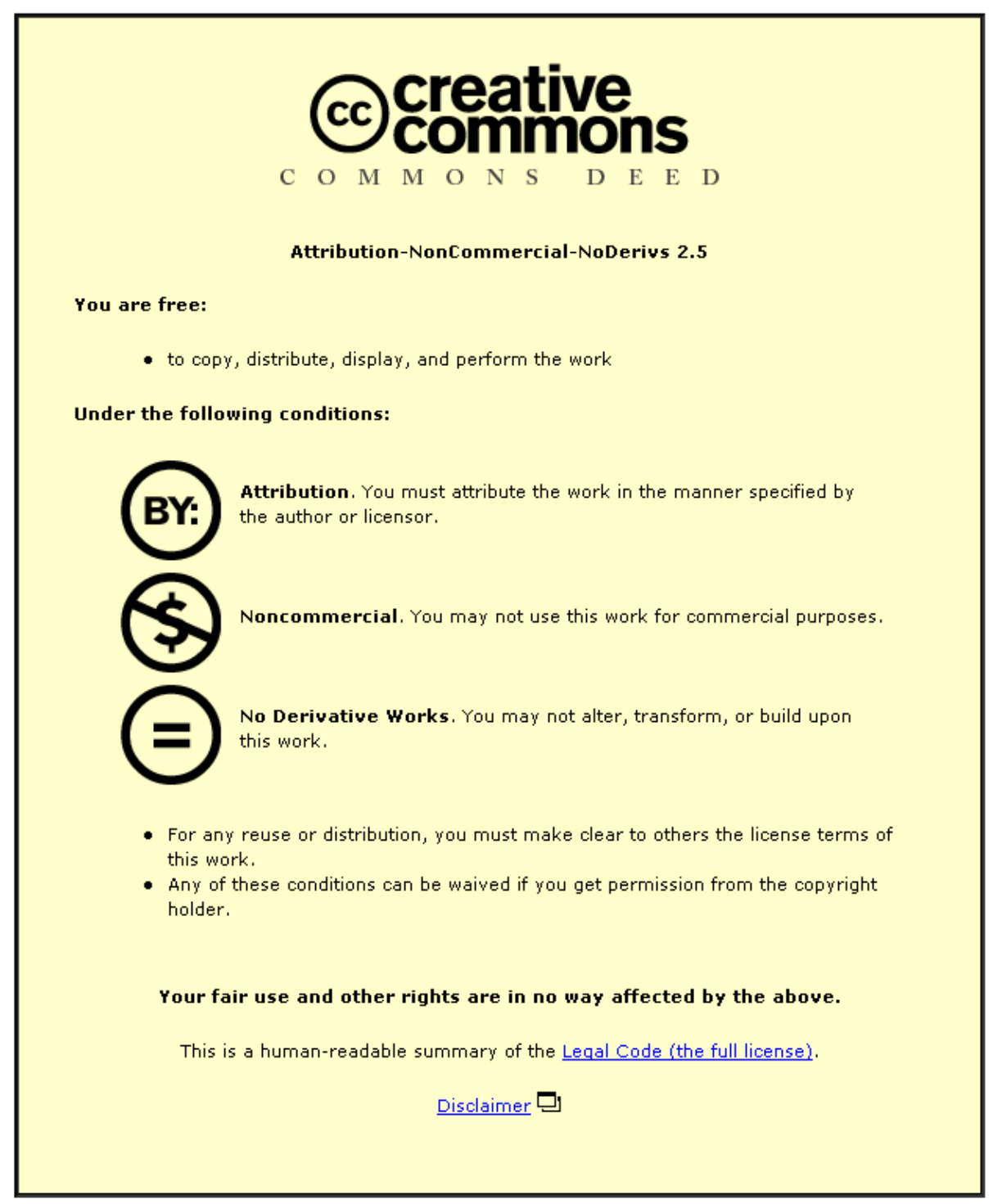

For the full text of this licence, please go to: http://creativecommons.org/licenses/by-nc-nd/2.5/ 


\title{
A NOVEL SINGLE LAG AUTO-CORRELATION MINIMIZATION (SLAM) ALGORITHM FOR BLIND ADAPTIVE CHANNEL SHORTENING
}

\author{
Rab Nawaz and Jonathon A. Chambers \\ Center of Digital Signal Processing, Cardiff School of Engineering \\ Queen's Buildings, PO Box 925, Cardiff CF24 0YF, Wales, UK \\ \{nawazr, chambersj@cf.ac.uk\}
}

\begin{abstract}
A blind adaptive channel shortening algorithm based on minimizing the sum of the squared autocorrelations (SAM) of the effective channel was recently proposed. We submit that identical channel shortening can be achieved by minimizing the square of only a single autocorrelation. Our proposed single lag autocorrelation minimization (SLAM) algorithm has, therefore, very low complexity and also it does not require, a priori, the knowledge of the length of the channel. We also constrain the autocorrelation minimization with a novel stopping criterion so that the shortening signal to noise ratio (SSNR) of the effective channel is not minimized by the autocorrelation minimization. The simulations have shown that SLAM achieves higher bit rates than SAM.
\end{abstract}

\section{INTRODUCTION}

In Multicarrier Modulation Systems (MCMs), such as Asymmetrical Digital Subscriber Line (ADSL) transceivers, a cyclic prefix $(\mathrm{CP})$ is used to combat Inter-symbol interference (ISI) and inter-channel interference (ICI) so that the equalization becomes trivial. The length of the $\mathrm{CP}$ should, at least, be equal to the order of the channel impulse response. The insertion of the $\mathrm{CP}$, however, reduces the bandwidth and power efficiency of the transmission by $v /(N+v)$, where $v$ is the length of $\mathrm{CP}$ and $N$ is the symbol duration without the $\mathrm{CP}$. In order to reduce this loss, a time domain equalizer (TEQ), usually an FIR filter, is inserted at the receiver front end. The convolution of the TEQ and the actual channel yields an effective channel of shorter length, which allows the use of a shorter $\mathrm{CP}$ and hence the loss in bandwidth and power is minimized, combating the ISI and ICI at the same time. Therefore the shortening effort in MCM systems and the improvement in $v /(N+v)$ factor become a trade-off. The TEQ design has been extensively studied in the literature. Most of the approaches have high complexity, require training or channel estimation and are non-adaptive [1]. A blind, adaptive channel shortener will always be better in more time varying environments and where less training is available [3]. Some emerging standards like, VDSL (Very High Speed DSL) standard do not even allow for training sequences for equalization [6]. The advantage in terms of bandwidth efficiency by doing channel shortening blindly becomes twofold, as no periodic transmission of a training signal is necessary.

A low complexity blind adaptive algorithm to design a TEQ, called Multicarrier Equalization by Restoration of Redundancy (MERRY) was proposed in [1] but its parameter updates are performed only once per symbol [2]. SAM [2], on the other hand, updates its coefficients more frequently than MERRY so that it can track the time variations within a symbol. Unlike MERRY, SAM is insensitive to synchronization errors and also converges much faster. The drawback with SAM is that it has a significantly higher computational complexity and it does not have any stopping criterion (explained in section 5) to freeze the channel shortener/TEQ when the shortening signal to noise ratio of the effective channel reaches close to the Maximum Shortening Signal to Noise Ratio (MSSNR) solution of [4]. We address these issues in this paper.

The paper is organized as follows. Section 2 gives the system model and the notations. Section 3 discuss the motivation for minimizing only one autocorrelation and gives the SLAM cost function. Section 4 discusses the steepest gradient-descent implementation of the SLAM algorithm. Section 5 discusses novel stopping criterion. Section 6 provides the comparative simulations between SLAM and SAM and section 7 concludes.

\section{SYSTEM MODEL}

The system model is shown in Fig. 1. The signal $x(n)$ is a white, zero-mean, wide-sense stationary (W.S.S), real and unit variance source sequence transmitted through the linear finite-impulse response (FIR) channel h. $v(n)$ is a zeromean, i.i.d., noise sequence uncorrelated with the source se- 
quence and has variance $\sigma_{v}^{2}$. The received signal $r(n)$ is

$$
r(n)=\sum_{k=0}^{L_{h}} h(k) x(n-k)+v(n)
$$

and $y(n)$, the output of the TEQ is given by

$$
y(n)=\sum_{k=0}^{L_{w}} w(k) r(n-k)=\mathbf{w}^{T} \mathbf{r}_{n}
$$

where $\mathbf{w}$ is the impulse response vector of the TEQ $\mathbf{w}=$ $\left[\begin{array}{lllll}w_{0} & w_{1} & w_{2} & \ldots & w_{L_{w}}\end{array}\right]^{T}$ and $\mathbf{r}_{n}=[r(n) r(n-1) \ldots r(n-$ $\left.\left.L_{w}\right)\right]^{T} . L_{h}, L_{c}$, and $L_{w}$ are the order of the channel, effective channel and the TEQ respectively. We denote $\mathbf{c}=\mathbf{h} \star \mathbf{w}$ as the shortened or effective channel. We also assume that $2 L_{c}<N$ holds, $N$ being the FFT size [2], which is quite a reasonable assumption in the case of ADSL.

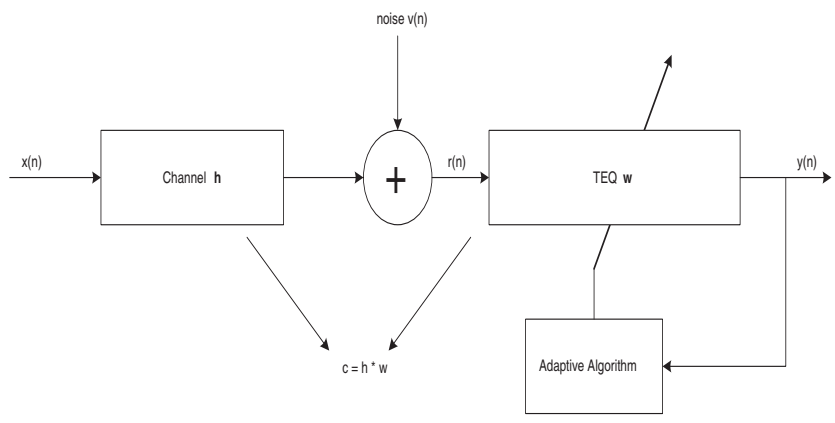

System model for blind adaptive channel shortening

Fig. 1. System model.

\section{SLAM}

SAM is based on the fact that for the effective channel $\mathbf{c}$ to have zero taps outside a window of size $(v+1)$, its autocorrelation values should be zero outside a window of size $(2 v+1)$. We submit that a single autocorrelation at a lag greater than the guard interval is a measure of the presence of the channel outside the desired guard interval. Therefore, minimizing only this single autocorrelation should give us the channel shortening effect. Now the autocorrelation sequence of the effective channel is given by

$$
R_{c c}(l)=\sum_{k=0}^{L_{c}} c(k) c(k-l)
$$

and for a shortened channel, it must satisfy

$$
R_{c c}(l)=0, l=v+1
$$

We, therefore, define a cost function, $J_{v+1}$, based upon minimizing the square of the auto-correlation of the effective channel at lag $l=v+1$, i.e.,

$$
J_{v+1}=\left|R_{c c}(l)\right|^{2}, l=v+1
$$

The trivial solution of $\mathbf{c}=0$, or $\mathbf{w}=0$, can be avoided by imposing a norm constraint on the effective channel response, for instance $\|\mathbf{c}\|_{2}^{2}=1$ or on the TEQ i.e., $\|\mathbf{w}\|_{2}^{2}=1$. The optimization problem can then be stated as

$$
\mathbf{w}^{\text {opt }}=\arg _{\mathbf{w}} \min _{\|\mathbf{c}\|_{2}^{2}=1} J_{v+1}
$$

The autocorrelation sequence of the output $y(n)$ is given by

$$
\begin{aligned}
R_{y y}(l) & =E[y(n) y(n-l)] \\
& =E\left[\left(\mathbf{c}^{T} \mathbf{x}_{n}+\mathbf{w}^{T} \mathbf{v}_{n}\right)\left(\mathbf{x}_{n-l}^{T} \mathbf{c}+\mathbf{v}_{n-l}^{T} \mathbf{w}\right)\right]
\end{aligned}
$$

given the stated conditions on $x(n)$ and $v(n)$ in section (2), equation (3) can be written as [2]

$$
R_{y y}(l)=R_{c c}(l)+\sigma_{v}^{2} R_{w w}(l)
$$

So that we can approximate our cost function in equation (2), denoted as $\hat{J}_{v+1}$,

$$
\begin{aligned}
\hat{J}_{v+1}= & \left|R_{y y}(l)\right|^{2} \\
= & \left|R_{c c}(l)\right|^{2}+2 \sigma_{v}^{2} R_{c c}(l) R_{w w}(l) \\
& +\sigma_{v}^{4}\left|R_{w w}(l)\right|^{2}
\end{aligned}
$$

where $l=v+1$. The second and third terms being added are very small due to their multiplication with $\sigma_{v}^{2}$ and $\sigma_{v}^{4}$ respectively. We presume that under practical SNR scenarios, we can drop the hat on $J_{v+1}$ so that $\hat{J}_{v+1} \cong J_{v+1}$. For this cost function we do not need the length of the channel $\mathbf{h}$ to determine $L_{c}$ as is required by SAM in [2]. Our algorithm only requires the output of the TEQ and is, in that sense, blind.

\section{ADAPTIVE ALGORITHM}

The steepest gradient-descent type algorithm to minimize $J_{v+1}$ is

$$
\mathbf{w}^{\text {new }}=\mathbf{w}^{\text {old }}-\mu \nabla_{\mathbf{w}}(E[y(n) y(n-l)])^{2}
$$

$l=v+1$. Here $\mu$ is the step size and $\nabla_{\mathbf{w}}$ is the gradient evaluated at $\mathbf{w}=\mathbf{w}^{\text {old }}$. We can use the auto-regressive (AR) estimates to implement the algorithm. Let

$$
\begin{aligned}
\mathbf{A}^{n} & =(1-\alpha) \mathbf{A}^{n-1}+\alpha y(n)\left[\begin{array}{c}
r(n-v-1) \\
\vdots \\
r\left(n-v-1-L_{w}\right)
\end{array}\right] \\
\mathbf{B}^{n} & =\mathbf{W A}^{n} \\
\mathbf{C}^{n} & =(1-\alpha) \mathbf{C}^{n-1}+\alpha y(n-v-1)\left[\begin{array}{c}
r(n) \\
\vdots \\
r\left(n-L_{w}\right)
\end{array}\right]
\end{aligned}
$$


where $0<\alpha<1$ is a forgetting factor and is a design parameter. $\mathbf{W}$ is the channel shortener row vector $\mathbf{W}=\mathbf{w}^{T}$. Using these AR estimates and equation (1), the update rule of equation (5) can be written as

$$
\begin{aligned}
\mathbf{w}^{n+1}= & \mathbf{w}^{n}-2 \mu\{E[y(n) y(n-l)]\} \\
& \cdot\left\{E\left[y(n) \mathbf{r}_{n-l}+y(n-l) \mathbf{r}_{n}\right]\right\} \\
\cong & \mathbf{w}^{n}-2 \mu\{\mathbf{B}\} \cdot\{\mathbf{A}+\mathbf{C}\}
\end{aligned}
$$

$\mathbf{w}$ is normalized at each iteration to implement the constraint $\|\mathbf{c}\|_{2}^{2}=1$. Equation (6) is the SLAM algorithm. The complexity of SAM is approximately $4 L_{w}\left(L_{c}-v\right)$ multiplications and additions each update, plus a division for renormalization. The complexity of SLAM is approximately $4 L_{w}$ which is about $1 / 500$ times that of SAM for ADSL downstream transmission environment parameters.

\section{STOPPING CRITERION}

In [2] the SAM algorithm achieves its maximum bit rate in the first few iterations and then decreases and later starts increasing the bit rate but at a very low rate (please see figure (4). This is identical with our SLAM algorithm. We need to stop the minimization of the autocorrelation before the point where SSNR of the shortened channel starts decreasing (giving a maximum possible achievable bit rate point). We propose a stopping criterion to freeze the channel shortening at the mentioned point. Specifically we examine the energy of the taps of the TEQ, except the center tap, and freeze the TEQ when the sum of the energy of these taps reaches a threshold. Simulations have shown the effectiveness of our stopping criterion. Our proposed stopping criterion does not add any extra computational complexity as we already are taking the norm of the TEQ at every iteration. Convergence analysis of SLAM is complicated by the nonquadratic nature of the cost function of eq. (4), nonetheless, on the basis of extensive simulation studies we have observed that the convergence properties of SLAM at least match those of SAM.

\section{SIMULATIONS}

The Matlab code available at [5] was used to simulate SLAM. The parameters chosen were that of ADSL standard downstream transmission. The CP was 32, the FFT size was 512, the TEQ length was 16 and the value of $\alpha$ was $1 / 100$. The channel was the test ADSL channel CSA loop 1 available at [6]. The noise power was set such that $\sigma_{x}^{2}\|\mathbf{h}\|^{2} / \sigma_{v}^{2}=40 \mathrm{~dB}$. A total of 75 symbols were used and 4-QAM signalling was used on all of the sub-carriers. One reason to keep the simulation parameters same as in [2] was to make a fair comparison. The initialization was single center spike and step size used for SLAM was 140. This step size enables to see the dropping of the achievable bit rate by SLAM within our simulated number of symbols. The threshold used was 0.0614. Once this threshold is achieved, we normalize the TEQ and freeze the channel shortening. SLAM is also compared with the maximum SSNR solution of [4]. Achievable bit rate for a fixed probability of error was chosen as the performance metric. The bit rate was determined based on

$$
R=\sum_{i=1}^{N_{f f t}} \log _{2}\left(1+S N R_{i} / \Gamma\right)
$$

The bit rate was determined using a 6-dB margin and a $4.2 \mathrm{~dB}$ coding gain. The details of how the bit rate is calculated are given in [7]. The remainder of the explanation relates to the figures mentioned individually.

Figure (2) shows the channel shortening effect while Figure (3) shows the TEQ designed. Figure (4) shows the achievable bit rate by SLAM at $40 \mathrm{~dB}$ SNR versus iteration number and demonstrate the effectiveness of the stopping criterion. SAM and SLAM both start to decrease the SSNR of the effective channel at some stage in the shortening. If we do not constrain them by some stopping criterion, both tend to decrease their achievable bit rates. In Figure (5) we show the achievable bit rate by SAM and by the SLAM algorithm for a range of SNRs. At each SNR, the bit rate was calculated for the settings of TEQ arrived at after 75 symbols. The improvement in bit rate by SLAM at such a drastically low complexity is evident, approaching the MSSNR solution of [4] for the whole range of SNRs.

\section{CONCLUSIONS}

SLAM has considerably lower complexity as compared to SAM. This makes it also suitable for shortening time varying channels, because the channel shortening can be performed online. SLAM does not need to know in advance the length of the channel. A novel stopping criterion which freezes the TEQ at the peak achievable bit rates has also been proposed. SLAM, with the stopping criterion, achieves better bit rates than SAM for a range of SNRs. Future work will involve channel shortening of the doubly selective OFDM channels.

Acknowledgement: The authors thank Dr. Richard K. Martin, the second author of [2], for his comments and the Matlab code. This work was funded by the Ministry of Science \& Technology, Govt. of Pakistan.

\section{REFERENCES}

[1] R. K. Martin, J. Balakrishnan, W. A. Sethars, and C. R. Johnson, Jr., "A blind, adaptive TEQ for multicarrier systems," IEEE Signal Processing Lett., vol. 9, pp. 341-343, Nov. 2002. 


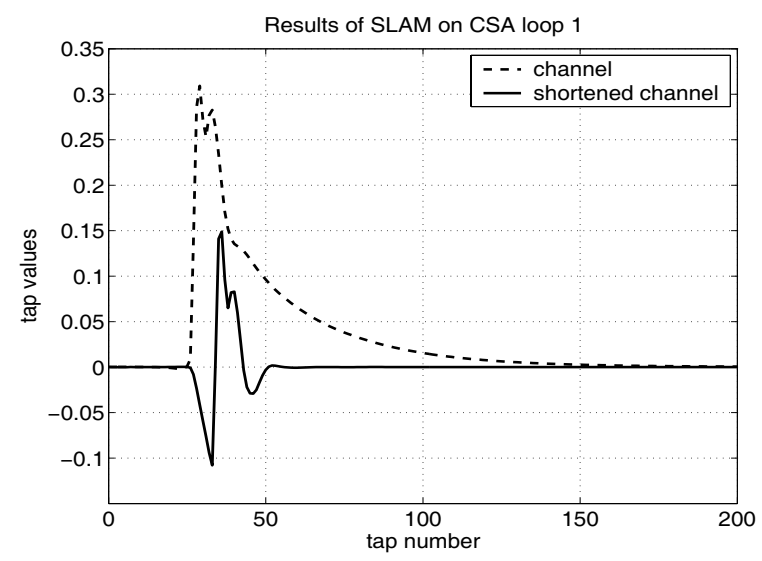

Fig. 2. Original and shortened channel.

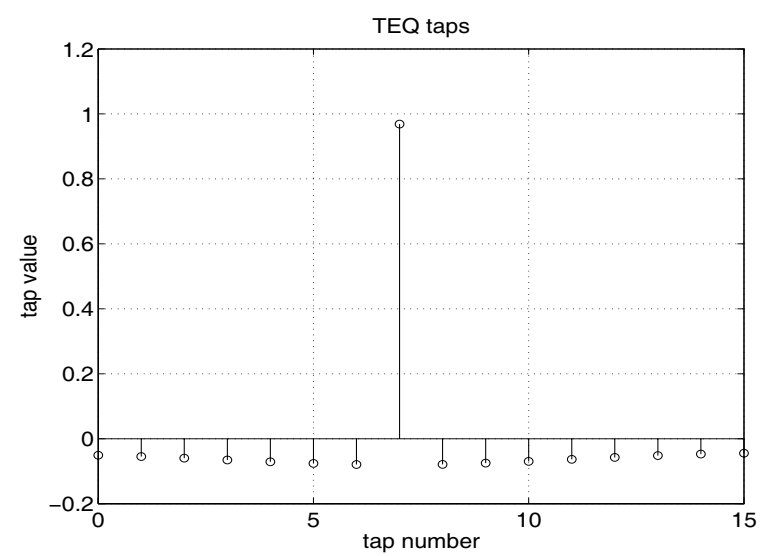

Fig. 3. TEQ or Channel shortener.

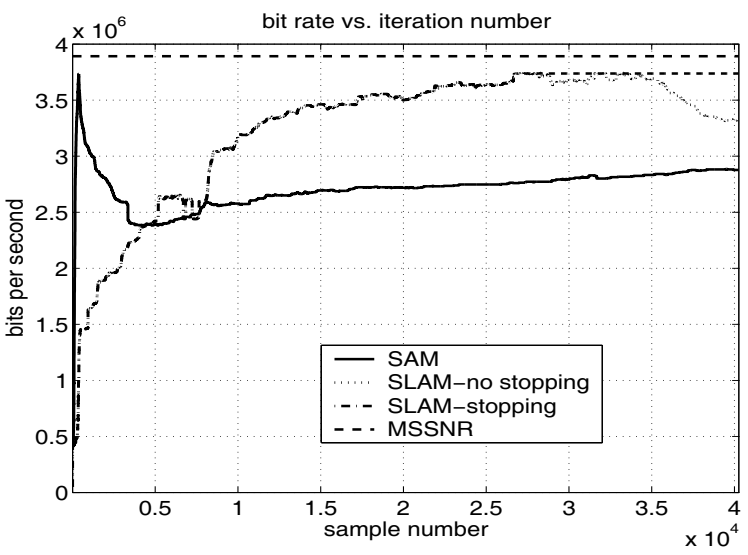

Fig. 4. Achievable bit rate versus iteration number at $40 \mathrm{~dB}$ SNR. Without stopping criterion applied, both SAM and SLAM start decreasing the bit rate.

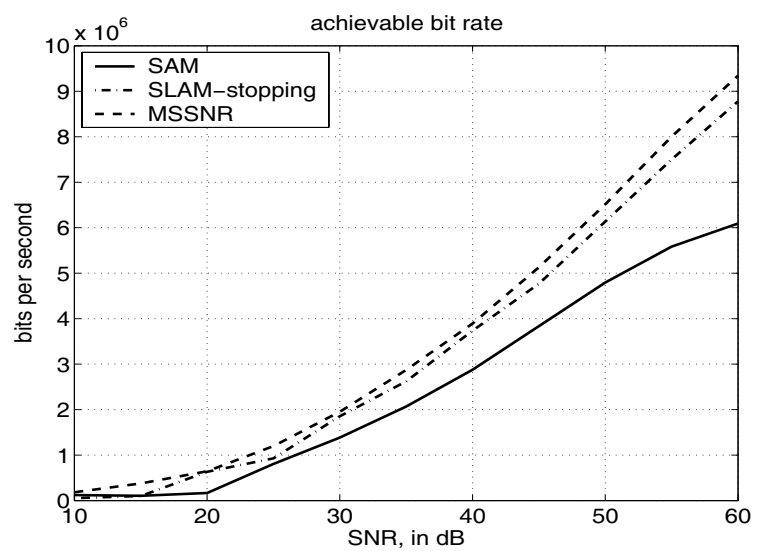

Fig. 5. Achievable bit rate versus SNR for SAM, SLAM and MSSNR.

[2] J. Balakrishnan, R. K. Martin and C. R. Johnson, Jr., "Blind, adaptive channel shortening by sum-squared auto-correlation minimization (SAM)," IEEE Trans. Signal Processing, vol. 51, no. 12, pp. 3086-3093, Dec. 2003.

[3] R. K. Martin, "Blind, adaptive equalization for multicarrier receivers," $\mathrm{Ph}$. D. Thesis, Cornell University, US 2004.

[4] P. J. W. Melsa, R. C. Younce, and C. E. Rohrs, "Impulse response shortening for discrete multitone transceivers," IEEE Trans. Commun., vol. 44, pp. 1662-1672, Dec. 1996.

[5] R. K. Martin. Matlab Code for Papers by R. K. Martin. [Online]. Available: http://bard.ece.cornell.edu/matlab/martin/index.html

[6] G. Arslan, M. Ding, B. Lu, Z. Shen, and B. L. Evans. TEQ Design Toolbox. Univ. Texas, Austin, TX. [Online] Available:http://www.ece.utexas.edu/ bevans/projects/ adsl/ dmtteq/dmtteq.html

[7] G. Arslan, B. L. Evans, and S. Kiaei, "Equalization for discrete multitone receivers to maximize bit rate," IEEE Trans. Signal Processing, vol. 49, pp. 31233135, Dec. 2001. 